\title{
Medição e controle do tempo de atravessamento em um sistema de manufatura
}

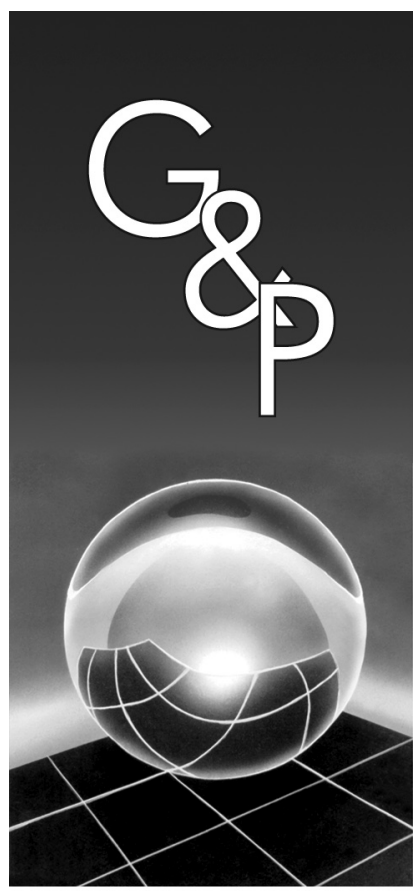

Miguel Afonso Sellitto

Cláudio Walter

\section{Resumo}

Este artigo apresenta um método para a medição do tempo de atravessamento e do inventário em processo em manufatura. A medição foi usada em processo de controle organizacional de manufatura, cuja variável controlada foi o tempo de atravessamento de ordens. Tal controle pode ser útil em estratégias de manufatura em que a competição é baseada no uso do tempo, a TBC (time-based competition). Apresentou-se o método de medição, que inclui elementos da teoria das filas, e considerações para a simplificação de arranjos produtivos de manufatura. Para testar e refinar o método, estudou-se um caso em manufatura calçadista. Foram coletados dados de remessas e obtido, por análise estatística e simulação computacional, o comportamento da variável aleatória tempo de atravessamento de remessas. Como a medição foi inferior ao objetivo de desempenho, partiu-se para o controle, fazendo um diagnóstico que apontou efeitos indesejáveis observados que foram endereçados por ações corretivas implementadas. Para verificar a eficácia das ações, nova coleta foi feita. Desta vez, o objetivo de desempenho das entregas foi atendido, fechando um ciclo de controle. Os resultados foram discutidos, chegando-se a conclusões e alternativas de continuidade.

Palavras-chave: Tempo de atravessamento em manufatura. Inventário em manufatura. Processos estocásticos em manufatura. Arranjos em manufatura.

\section{Introdução}

Medição e controle de tempo de atravessamento em cadeias produtivas e logísticas podem ser úteis na formulação e execução de estratégias de manufatura para competição baseadas em uso do tempo, a time-based competition (TBC - competição baseada em tempo) (STALK; HOUT, 1990). Para os autores, TBC é mais do que atender datas devidas; é gerenciar o tempo como um recurso produtivo limitado, identificando e reduzindo atividades de longa duração.

Um modo de gerenciar o tempo como recurso produtivo é medir e controlar tempos de atravessamento e inventários, grandezas que representam medidas de tempo de espera e de tamanho das filas na manufatura. O tempo de atravessamento médio no sistema produtivo reflete as consequiências das práticas gerenciais da organização, principalmente a maneira como esta trata as variabilidades. Para reduzir o tempo de atravessamento, limita-se o inventário ao mínimo necessário para evitar a ociosidade que pode surgir por falta de carga na manufatura (HEARD; PLOSSL, 1984). O uso estratégico do tempo contribui para objetivos de manufatura, tais como tornar menores e mais confiáveis os prazos de entrega (SLACK, 1993).

O objetivo deste artigo é propor e testar um método para medição e eventual controle do tempo de atravessamento em operações de manufatura, que se valham de ordens de fabricação. Por ser uma grandeza correlacionada com o tempo de atravessamento, o inventário em processo também resulta afetado. Por controle, entende-se um procedimento no qual, por um conveniente modelo, mede-se uma grandeza, compara-se a medição com um objetivo e, se houver diferença, dentre um conjunto de ações possíveis, uma inteligência de governança escolhe aquelas mais indicadas para reduzir a diferença. Executadas as ações, aplica-se novamente o modelo de medição e conclui-se se houve eficácia no conjunto de ações tomadas. $\mathrm{O}$ método de pesquisa foi o estudo de caso único, do tipo exploratório.

Algumas referências trazem contribuições relacionadas com o objetivo da pesquisa. Bartezzaghi et al. (1994) apresentam um modelo de cálculo de tempos de atravessamento em processo para reengenharia. Wacker (1996) apresenta um modelo para manufatura, que 
examina um produto por vez. Vollmann et al. (2006) apresentam um método para cálculo do tempo de atravessamento apriorístico. Pan e Yang (2002) e Bem-Daya e Hariga (2003) relacionam controle de tempo de atravessamento e inventário. Bechte (1994), Wiendahl e Breithaupt (2001), Breithaupt et al. (2002) e a obra de síntese Wiendahl (1995) apresentam o WLC (workload control - controle pela carga de trabalho), que controla a liberação de ordens para manufatura, mantendo o inventário abaixo de um limite e, por consequiência, o tempo de atravessamento ao redor de um objetivo.

O restante do artigo está organizado em: (i) descrição do modelo de medição usado; (ii) representação e simplificação de arranjos em manufatura; (iii) medição de tempo de atravessamento em operação de manufatura calçadista; (iv) ações de controle do tempo de atravessamento; e (v) considerações finais.

\section{Medição de tempo de atravessamento e inventário}

O termo tempo de atravessamento tem servido como tradução para a expressão lead-time, usada na literatura internacional para endereçar ao menos duas grandezas: (i) em suprimentos, tempos relacionados a reposições de materiais; e (ii) em produção, tempos relacionados ao atendimento de demandas de clientes. Este artigo trata da segunda grandeza.

Tempo de atravessamento em produção é a medida do tempo que um sistema produtivo gasta para transformar matérias-primas em produtos acabados disponíveis para uso (TUBINO, 1990; CORREA et al., 2001). MacCarthy e Fernandes (2000) falam em tempo de resposta, composto de tempo de suprimento (SL), de produção (PL) e de distribuição (DL). Neste artigo, o tempo de atravessamento considerado é a soma dos dois primeiros tempos, que foi chamado de tempo de atravessamento de ordens de fabricação, o tempo que a manufatura gasta, desde o aceite comercial da ordem, que dispara a compra e coleta de materiais, até quando o produto estiver disponível para o transporte até o cliente. Neste tempo, estão presentes os seguintes componentes: (i) emissão da ordem, compra e coleta de materiais e chegada ao chão de fábrica; (ii) transporte até a primeira atividade; (iii) espera em fila e até atingir o tamanho do lote; (iv) processamento (setup e operações); (v) inspeção e eventual retrabalho; (vi) transporte até a próxima atividade; e (vii) recorrência até a última atividade. Wacker (1996) acrescenta tempos de interrupção por falta de materiais e manutenção de emergência.

Tempos de atravessamento de ordens podem ser calculados, a priori, para uso em sistemas de controle de produção, tal como MRP's, por estimativas fixas calculadas conforme a técnica de programação e a prioridade da ordem (BERTRAND et al., 1990). Também podem ser medidos, a posteriori, com dados de campo, por variáveis aleatórias (SELLITTO, 2005). Ambos podem ser controlados e reduzidos (PAN; YANG, 2002), mas, para este artigo, interessam tempos a posteriori, cuja variabilidade é causada pela variabilidade intrínseca dos componentes de tempo e pela variação no esforço requerido pela ordem.

O tempo de atravessamento simples TL de uma ordem em um centro de trabalho é obtido diminuindo a data de conclusão no centro anterior da data de conclusão do centro atual. $\mathrm{O}$ tempo de atravessamento ponderado $\mathrm{TL}_{\mathrm{w}}$ multiplica o TL por uma unidade de valor, proporcional ao esforço de manufatura requisitado pela ordem, tal como o número de peças, a tonelagem processada ou o número de horas padrão da ordem: por exemplo, quanto mais tempo foi usado e quanto maior for a quantidade produzida, mais esforço de manufatura foi requerido. Nas Equações 1 e 2 surgem as expressões para TL e $\mathrm{TL}_{\mathrm{w}}$ médios $\left(\mathrm{TL}_{\mathrm{m}}\right.$ e $\left.\mathrm{TL}_{\mathrm{mw}}\right)$, em que $\mathrm{TL}_{\mathrm{i}}$ e $\mathrm{Q}_{\mathrm{i}}$ são, respectivamente, os tempos de atravessamento e a quantidade processada na ordem i (WIENDAHL, 1995). O tempo padrão TO da ordem também pode ser usado.

$$
\begin{aligned}
\mathrm{TL}_{\mathrm{m}} & =\frac{\sum_{\mathrm{i}=1}^{\mathrm{n}} \mathrm{TL}_{\mathrm{i}}}{\mathrm{n}} \\
\mathrm{TL}_{\mathrm{mw}} & =\frac{\sum_{\mathrm{i}=1}^{\mathrm{n}} \mathrm{TL}_{\mathrm{i}} \cdot \mathrm{Q}_{\mathrm{i}}}{\sum_{\mathrm{i}=1}^{\mathrm{n}} \mathrm{Q}_{\mathrm{i}}}
\end{aligned}
$$

Wiendahl (1995) dá uma interpretação física a um $\mathrm{TL}_{\mathrm{wmm}}$, ou seja, é o valor esperado para o tempo que uma peça ou uma atividade prevista para durar uma hora leva para atravessar um centro de trabalho. Sellitto (2005) apresenta um método para escolher entre o $\mathrm{TL}_{\mathrm{m}}$ e um $\mathrm{TL}_{\mathrm{wm}}$ para ações de controle estratégico. Aplica-se uma anova aos valores obtidos e se ao nível de significância de $5 \%$ não houver diferença, usa-se por parcimônia de cálculo o $\mathrm{TL}_{\mathrm{m}}$.

Associado ao tempo de atravessamento, um método para a medição do inventário é usar uma representação gráfica do processo de fila, ou seja, o diagrama de resultados (Figura 1).

No diagrama, os traçados acumulam as entradas e as saídas de valor no período P. A entrada inicial é a quantidade de trabalho em fila de espera no início do período, a partir da qual acumulam-se entradas, nas datas em que ocorrem. A saída é traçada pela adição das ordens completadas, na mesma unidade da entrada, nas respectivas datas de saída. INP e OUT representam, respectivamente, o total de trabalho admitido e concluído durante P. A área hachurada representa o esforço de manufatura, proporcional à quantidade de peças e ao tempo de atra- 
vessamento das ordens. Em t, a distância vertical $\mathrm{I}(\mathrm{t})$ é o inventário instantâneo e a distância horizontal TL(t) é o tempo de atravessamento instantâneo. Como as ordens são discretas, trabalha-se com valores médios do tempo de atravessamento e do inventário, representados pelas distâncias médias entre os traçados de entrada e de saída no período. Pode-se verificar o cálculo do inventário pela lei de Little (Equação 3), na qual $\mathrm{TL}_{\mathrm{m}}$ é o tempo de atravessamento médio, $\mathrm{I}_{\mathrm{m}}$ é o inventário médio e $\mathrm{P}_{\mathrm{m}}$ é o desempenho médio, em unidades de valor por unidade de tempo (KLEINROCK, 1975).

$$
\mathrm{TL}_{\mathrm{m}}=\frac{\mathrm{I}_{\mathrm{m}}}{\mathrm{P}_{\mathrm{m}}}
$$

\section{Controle de produção em arranjos de manufatura}

Processos de controle em organizações exigem planejamento inicial, execução, medição do desempenho, comparação com objetivos e tomada de ações de correção. Especificamente, o controle de produção exige: (i) arranjo prévio dos recursos produtivos, tais como pessoal, equipamentos e sistemas de informação; (ii) planos de fabricação; (iii) execução dos planos; e (iv) à luz da comparação entre desempenho medido e objetivos, coordenação dos recursos de manufatura para

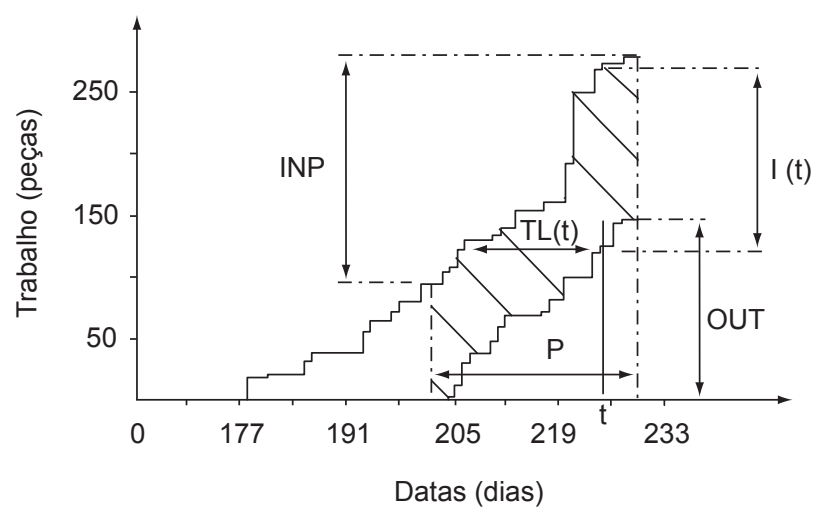

Figura 1. Diagrama de resultados (fonte: WIENDAHL, 1995) ações de correção dos planos. São necessários, além de sistema de informações, planos de ação principais e alternativos, estes requisitados se o resultado do curso de ação original não cumprir os objetivos da manufatura, em ajuste contínuo (BONNEY, 2000; SELLITTO, 2005). Stekelenborg e Kornelius (1994) tipificam controles de produção: controle da demanda, que usa como regulador o ritmo de liberação de ordens para a manufatura, e controle de capacidade, que usa como regulador a modificação da capacidade operacional.

O controle de produção pode ser: (i) estrito, se as ações são locais e baseadas no valor instantâneo de uma variável local; e (ii) amplo, se as ações são estruturais e baseadas na distribuição de probabilidade de uma variável global (SELLITTO, 2005). Também pode ser exercido em nível: (i) operacional, controlando o desempenho das ordens individuais de fabricação; e (ii) planejamento, controlando o desempenho médio do conjunto das ordens. O controle pode ser por malha fechada, desde que se definam variáveis de controle. No caso operacional, usa-se o valor instantâneo e para planejamento, o valor médio da variável (WIENDAHL; PETERMANN, 1994). Neste artigo, além do valor médio, usaram-se intervalos de confiança para a variável de controle.

Abordagens baseadas na malha fechada de controle têm sido desenvolvidas para o controle de produção, tais como o elo de realimentação dos sistemas MRP II, que estimula reações do sistema ao comparar os resultados medidos com o objetivo de referência. Segundo Wiendahl e Breithaupt (2001) e Wiendahl e Breithaupt (1999), uma fraqueza observada nestes sistemas foi a falta de clareza sobre quais são as variáveis de referência e controle e sobre a relação entre causas e efeitos nas ações, devido ao determinismo assumido em grandezas tais como o tempo de atravessamento e o desempenho de produção.

Neste artigo, controlou-se o desempenho da manufatura por malha fechada, calculando intervalos de confiança do tempo de atravessamento de ordens. A lógica de controle foi a da Figura 2. Havendo um objetivo para a grandeza e um modelo para a sua medição, estes são comparados e um sinal de erro é gerado. Se o erro for significativo, deve motivar a proposição, priorização e execução de

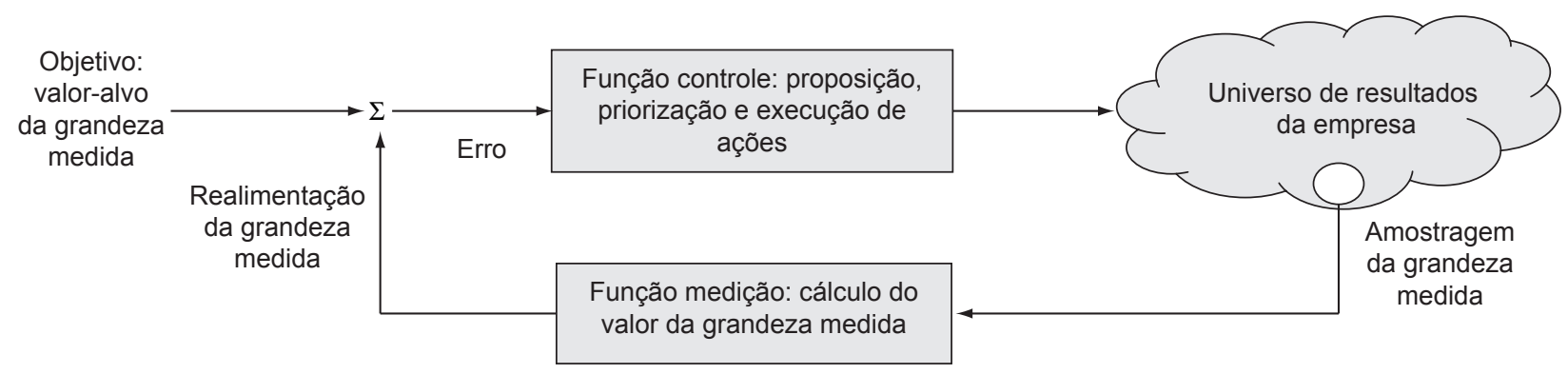

Figura 2. Controle organizacional em malha fechada (Fonte: SELLITTO, 2005). 
ações de controle, cujo objetivo seja reduzir o erro. Não é função do modelo de medição propor ações: esta deve ser feita pela inteligência que governa a organização. A medição apenas informa o quanto as ações reduziram o erro observado.

Se a ordem de fabricação deve passar por mais de um centro de trabalho, pode ser interessante agrupar centros afins, formando arranjos de manufatura. Para tanto, é conveniente lançar mão de algum tipo de classificação de sistemas produtivos. MacCarthy e Fernandes (2000) apresentam uma compreensiva revisão bibliográfica sobre classificação de sistemas produtivos e subsistemas de manufatura, na qual surgem classes de sistemas baseadas em diversos atributos. Os autores propuseram uma sistemática de classificação, baseada em quatro grandes caracterizações (geral, produto, processo e montagem), que se desdobram em doze variáveis, dentre elas o tempo de resposta, o nível de repetição, o número de produtos e o tipo de pulmão, as quais compareceram na presente pesquisa. Para o objetivo de pesquisa, porém, é suficiente considerar arranjos em divergências e convergências e a classificação VAT, citada pelos autores como apresentada por Frizelle (1989).

Arranjos de manufatura podem ser: (i) divergentes, se poucos materiais se transformam em muitos produtos acabados; (ii) convergentes, se muitos materiais se transformam em poucos produtos acabados; e (iii) processos, se as transformações são seriais, sem convergências ou divergências. Arranjos podem ser classificados em estruturas V, A, T e X. Em arranjos V, os materiais processados se abrem em caminhos divergentes; em A, se fecham em caminhos convergentes; em T, seguem um caminho serial até a última operação, da qual nascem diversos produtos; e em X, há uma combinação entre V e A (BURBIDGE, 1978; COX; SPENCER, 1998; SLACK et al., 1997). Sellitto (2005) cita o arranjo I, uma forma simplificada para análise, na qual as atividades se agrupam em um caminho serial que não se divide ao fim. Em duas atividades em série, inventários e tempos de atravessamento podem ser somados. Uma convergência pode ser transformada em uma série, somando-se o inventário dos processos a montante e tomando-se como tempo de atravessamento o maior tempo individual de ramo a montante. Uma divergência pode ser dividida em caminhos seriais. O inventário e o tempo de atravessamento dos processos resultantes são obtidos segregando-se as operações do processo focal conforme seu destino. A Figura 3 ilustra a simplificação de arranjos em formato I.

\section{A pesquisa}

A questão de pesquisa foi a seguinte: como medir tempo de atravessamento em manufatura e usar a medição no processo de controle desta grandeza? O objetivo de pesquisa foi testar em campo um método proposto por
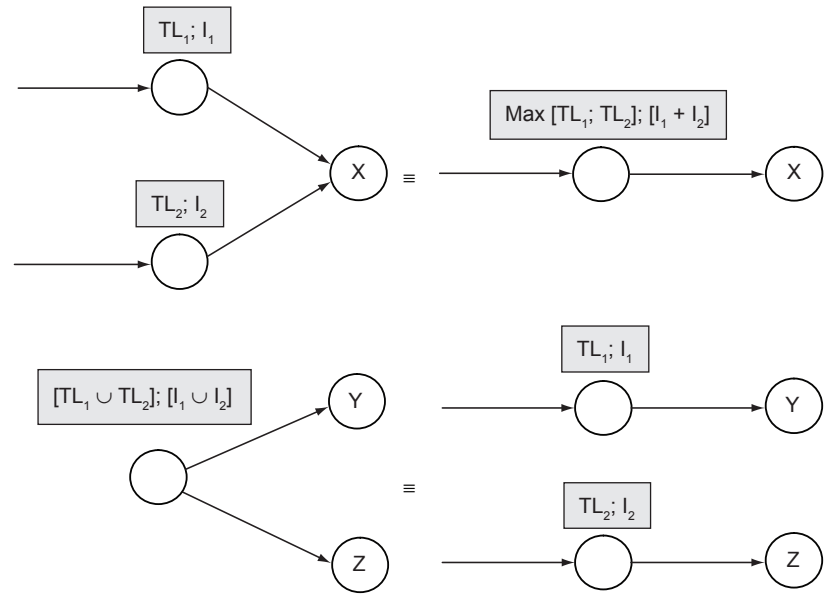

Figura 3. Transformações simplificadoras em arranjos produtivos.

estudos teóricos para a medição. A medição foi expressa sob a forma de variável contínua, associada ao valor médio da variável, e um intervalo de confiança, que incluiu a variabilidade. O método de pesquisa possível foi o estudo de caso, conduzido em um sistema de manufatura controlado por remessas a clientes.

O método do caso examina em profundidade um fenômeno, não sendo necessário nem desejável separá-lo do ambiente. O caso pode ser exploratório, se levanta hipóteses para estudos futuros; descritivo, se procura e testa associações entre variáveis definidas em explorações; ou explanatório, se apresenta explicações plausíveis para associações descritas (YIN, 2001). Casos podem contribuir para uma teoria de cinco modos: (i) oferecer, para outros estudos, descrições profundas de objetos; (ii) interpretar regularidades observadas como evidências de postulados mais gerais, ainda não verificados; (iii) criar deliberadamente uma situação para testar uma idéia; (iv) fazer uma sondagem plausível de uma teoria proposta; e (v) o caso crucial, que apóia ou refuta a teoria. Os objetivos são, em graduação, a exploração, a geração e o teste de teorias (ECKSTEIN, 1975, apud ROESCH, 1999).

Entende-se que o presente caso seja descritivo, já que é mais do que um levantamento de hipóteses para estudos futuros, aproximando-se de uma descrição de associações e relações entre variáveis conhecidas do objeto de estudo. Não chega a ser explanatório, pois não se oferecem explicações para a situação de todas as variáveis estudadas, apenas de algumas delas. Entende-se que a contribuição seja do terceiro tipo, pois foi construída uma situação para testar uma idéia.

O método de trabalho foi: (i) escolha da empresa, estudo e agregação das operações de manufatura; (ii) coleta de dados de quantidades, tempos-padrão e datas de início e conclusão das ordens de fabricação; (iii) por técnica estatística, diagrama de resultados e simulação computacional, cálculo de tempos de atravessamento e 
inventários; (iv) comparação com objetivo, proposição e implementação de ações de controle do tempo de atravessamento; e (v) análise e discussão do resultado, refinamento e conclusão.

\subsection{0 caso}

A manufatura escolhida foi uma montadora calçadista que usa fornecedores e terceiros nas operações de organização, fabricação, logística de transporte e armazenagem, em instalações próprias e de fornecedores espalhados em cerca de cem quilômetros de raio. Há variedade no número e no tipo dos materiais requeridos e os centros de trabalho transportam, fazem inspeções de entrada, armazenam, separam, processam, fazem inspeções de saída e dispõem sobras. Como as operações são espalhadas geograficamente, inspeções visuais em busca de inventário excessivo contam menos do que informações integradas em meio eletrônico.

O sistema estudado se centraliza na montadora. Fechado um pedido de cliente, composto de vários modelos e numerações, o PCP, por MRP, define os materiais requeridos e emite as ordens de compras. Recebidos os materiais e formado o lote inicial, o PCP libera para cada modelo uma ordem de fabricação que agrega as quantidades requisitadas por vários pedidos. As partes das ordens que compõem o pedido formam a remessa, de mesma quantidade do pedido. Uma ordem, portanto, participa de várias remessas. Para rastreamento, as remessas são recompostas pelo sistema de informação ao fim das atividades parciais.

Os intervalos entre liberações para fabricação são aleatórios. As ordens se iniciam na fábrica no setor de corte, vão à rede de ateliês, retornam à linha de montagem e se encerram na embalagem e expedição. $\mathrm{O}$ sistema de informações as rastreia e informa, em forma de remessas, dados de transferências entre atividades.

Para o objetivo de pesquisa, as operações foram agregadas em formato I: (i) liberação, composta das tarefas administrativas de dividir os pedidos de clientes em ordens de fabricação, requisitar, comprar, receber, organizar, agregar em lotes, contratar serviços de ateliês, seqüenciar as atividades e agregar as remessas; (ii) corte, na fábrica; (iii) costura, em ateliês da região, que formam um conjunto de processadores em paralelo; (iv) montagem, que compõe materiais recebidos de ateliês com outros materiais, como adesivos; e (v) expedição, composta das tarefas de inspeção dos pares, embalamento, recomposição das remessas, inspeção final de quantidades e conteúdo e despacho. Um grafo correspondente ao modelo de análise do sistema manufatureiro e é apresentado na Figura 4.

As informações das transferências de quinze remessas foram obtidas do sistema de informação da empresa. A contagem do tempo de atravessamento se inicia ao estar o material inspecionado e preparado no centro de

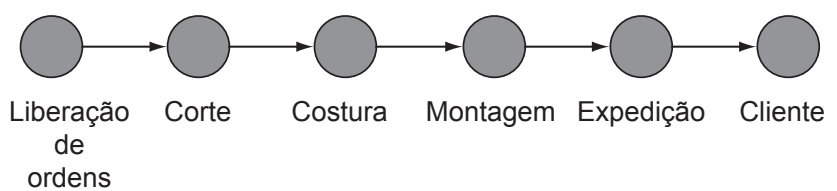

Figura 4. Grafo representativo do arranjo de manufatura em estudo.

trabalho anterior para o transporte interno, somando os tempos de transporte de chegada, de espera em fila e de processamento.

A Tabela 1 apresenta as quantidades totais de pares de calçados da remessa, o tempo-padrão previsto TO e os tempos de atravessamento simples TL em cada atividade e total. Apresenta também o valor esperado $\mu$, o desvio padrão $\sigma$ e o coeficiente de variação $\mathrm{cv}=\sigma / \mu$ para os tempos de atravessamento. Pode haver ultrapassagens: a remessa 5, por exemplo, é a quinta a iniciar e a primeira a encerrar. Uma expressiva parte do tempo é consumida nos vários tipos de esperas. A primeira remessa, por exemplo, apresenta um tempo de atravessamento total de 46 dias e meio e um tempo-padrão previsto de sete dias. A média dos TO é de 9,63 dias e dos TL é de 55,17 dias. A espera média é de 45,64 dias, o que corresponde a $[45,64 / 55,17]=82,7 \%$ do tempo consumido na manufatura.

$\mathrm{Na}$ Tabela 2, apresentam-se os tempos de atravessamento ponderados pela quantidade de pares $\mathrm{TL}_{\mathrm{w}}$, resultantes da multiplicação dos tempos de atravessamento simples TL pela quantidade de pares Q da remessa. Os tempos de atravessamento médios $\mathrm{TL}_{\mathrm{wm}}$ são calculados dividindo-se a soma dos $\mathrm{TL}_{\mathrm{w}}$ pela quantidade total de pares produzidos, segundo a Equação 2. O coeficiente de variação cv é obtido dividindo-se o desvio padrão dos $\mathrm{TL}_{\mathrm{w}}$ pela média. Uma tabela similar ponderada pelos tempos operacionais TO também foi obtida. Os resultados de tempos de atravessamento médios são resumidos na Tabela 3: $\mathrm{TL}_{\mathrm{m}}$ simples, $\mathrm{TL}_{\mathrm{Q}}$ e $\mathrm{TL}_{\mathrm{TO}}$. Uma anova apontou $\operatorname{com} \alpha=5 \%$ que a mudança de critério de cálculo não afeta o valor esperado do resultado. Por parcimônia, adota-se o tempo de atravessamento simples TL, segundo o método apontado em Sellitto (2005) e já mencionado.

Traçou-se a seguir o diagrama de resultados para o formato I resultante. O traçado aleatório foi substituído por retas obtidas pelo método dos mínimos quadrados. A substituição oferece uma expressão analítica para o cálculo de tempos de atravessamento e inventários e é avaliada pelo coeficiente de determinação $\mathrm{R}^{2}$ : quanto mais próximo de 1 , mais eficaz a substituição. Na Tabela 4, apresentam-se as equações lineares substitutas, os $\mathrm{R}^{2}$ e o aumento de inventário no período. Na Figura 5, surgem as retas. Até a quarta reta, as declividades são parecidas, o que indica equilíbrio reforçado pelos coeficientes angulares próximos. A partir 
Tabela 1. Tempos de atravessamento simples das remessas, em dias (Fonte: sistema de informações da empresa).

\begin{tabular}{ccccccccccc}
\hline $\begin{array}{c}\text { Remessa } \\
\mathbf{n}^{\mathbf{0}}\end{array}$ & $\begin{array}{c}\text { Data } \\
\text { início }\end{array}$ & $\begin{array}{c}\text { Quantidade } \\
\text { pares Q }\end{array}$ & $\begin{array}{c}\text { TO } \\
\text { total }\end{array}$ & $\begin{array}{c}\text { TL } \\
\text { liberação }\end{array}$ & $\begin{array}{c}\text { TL } \\
\text { corte }\end{array}$ & $\begin{array}{c}\text { TL } \\
\text { costura }\end{array}$ & $\begin{array}{c}\text { TL } \\
\text { montagem }\end{array}$ & $\begin{array}{c}\text { TL } \\
\text { expedição }\end{array}$ & $\begin{array}{c}\text { TL } \\
\text { total }\end{array}$ \\
\hline 1 & 0 & 10.100 & 7,00 & 11,00 & 1,00 & 14,00 & 8,50 & 12,00 & 46,50 \\
2 & 1,5 & 19.400 & 13,50 & 12,90 & 15,00 & 16,00 & 9,50 & 7,50 & 60,90 \\
3 & 3,0 & 11.400 & 7,00 & 13,80 & 3,70 & 15,00 & 3,50 & 14,00 & 50,00 \\
4 & 5,5 & 16.100 & 10,75 & 16,20 & 16,00 & 16,00 & 8,90 & 16,00 & 73,10 \\
5 & 10,5 & 10.200 & 8,50 & 8,90 & 1,80 & 5,80 & 1,80 & 4,00 & 22,30 \\
6 & 12,0 & 18.500 & 13,00 & 4,30 & 10,00 & 18,70 & 14,50 & 13,30 & 60,80 \\
7 & 21,0 & 14.300 & 10,75 & 19,40 & 6,00 & 18,70 & 8,30 & 5,50 & 57,90 \\
8 & 25,0 & 10.700 & 8,50 & 11,00 & 14,00 & 12,00 & 13,40 & 8,50 & 58,90 \\
9 & 27,0 & 19.600 & 13,50 & 8,14 & 18,00 & 8,00 & 16,30 & 14,80 & 65,24 \\
10 & 28,5 & 11.000 & 7,50 & 21,00 & 5,00 & 16,00 & 7,40 & 4,00 & 53,40 \\
11 & 30,5 & 7.300 & 4,75 & 5,67 & 13,00 & 19,00 & 2,00 & 8,70 \\
12 & 31,0 & 14.700 & 9,75 & 15,60 & 9,00 & 15,00 & 5,00 & 5,50 & 5,37 \\
13 & 36,0 & 15.500 & 10,75 & 13,10 & 8,00 & 10,00 & 19,00 & 9,00 \\
14 & 40,5 & 11.400 & 8,00 & 14,10 & 3,00 & 19,00 & 16,00 & 2,00 \\
15 & 43,0 & 16.600 & 11,25 & 14,00 & 7,00 & 18,00 & 13,80 & 14,00 & 59,10 \\
$\mu$ & & 13.787 & 9,63 & 12,61 & 8,70 & 14,75 & 9,86 & 9,25 & 54,10 \\
$\sigma$ & & 3.799 & 2,61 & 4,63 & 5,45 & 4,13 & 5,44 & 4,51 & 11,71 \\
$c v$ & & 0,28 & 0,27 & 0,37 & 0,63 & 0,28 & 0,55 & 0,49 & 0,21 \\
\hline
\end{tabular}

Tabela 2. Tempos de atravessamento ponderados por unidades produzidas.

\begin{tabular}{|c|c|c|c|c|c|c|c|}
\hline Remessa $\mathbf{n}^{\circ}$ & $\begin{array}{c}\text { Quantidade } \\
\text { pares Q }\end{array}$ & $\begin{array}{c}\text { TL }_{\text {wQ }} \\
\text { liberação }\end{array}$ & $\begin{array}{l}\mathrm{TL}_{\mathrm{wQ}} \\
\text { corte }\end{array}$ & $\begin{array}{c}\mathrm{TL}_{\mathrm{wQ}} \\
\text { costura }\end{array}$ & $\begin{array}{c}\mathrm{TL}_{\mathrm{wQ}} \\
\text { montagem }\end{array}$ & $\begin{array}{c}\mathrm{TL}_{\mathrm{wQ}} \\
\text { expedição }\end{array}$ & $\begin{array}{l}\mathrm{TL}_{\mathrm{wQ}} \\
\text { total }\end{array}$ \\
\hline 1 & 10.100 & 111.100 & 10.100 & 141.400 & 85.850 & 121.200 & 469.650 \\
\hline 2 & 19.400 & 250.260 & 291.000 & 310.400 & 184.300 & 145.500 & 1.181 .460 \\
\hline 3 & 11.400 & 157.320 & 42.180 & 171.000 & 39.900 & 159.600 & 570.000 \\
\hline 4 & 16.100 & 260.820 & 257.600 & 257.600 & 143.290 & 257.600 & 1.176 .910 \\
\hline 5 & 10.200 & 90.780 & 18.360 & 59.160 & 18.360 & 40.800 & 227.460 \\
\hline 6 & 18.500 & 79.550 & 185.000 & 345.950 & 268.250 & 246.050 & 1.124 .800 \\
\hline 7 & 14.300 & 277.420 & 85.800 & 267.410 & 118.690 & 78.650 & 827.970 \\
\hline 8 & 10.700 & 117.700 & 149.800 & 128.400 & 143.380 & 90.950 & 630.230 \\
\hline 9 & 19.600 & 159.606 & 352.800 & 156.800 & 319.480 & 290.080 & 1.278 .766 \\
\hline 10 & 11.000 & 231.000 & 55.000 & 176.000 & 81.400 & 44.000 & 587.400 \\
\hline 11 & 7.300 & 41.391 & 94.900 & 138.700 & 14.600 & 63.510 & 353.101 \\
\hline 12 & 14.700 & 229.320 & 132.300 & 220.500 & 73.500 & 80.850 & 736.470 \\
\hline 13 & 15.500 & 203.050 & 124.000 & 155.000 & 294.500 & 139.500 & 916.050 \\
\hline 14 & 11.400 & 160.740 & 34.200 & 216.600 & 182.400 & 22.800 & 616.740 \\
\hline 15 & 16.600 & 232.400 & 116.200 & 298.800 & 229.080 & 232.400 & 1.108 .880 \\
\hline $\mathrm{TL}_{\mathrm{wm}}$ & 13.787 & 12,58 & 9,43 & 14,72 & 10,62 & 9,74 & 57,09 \\
\hline$\sigma$ & 3.799 & 73.595 & 102.828 & 79.796 & 98.346 & 86.487 & 330.651 \\
\hline $\mathrm{cv}$ & 0,28 & 0,42 & 0,79 & 0,39 & 0,67 & 0,64 & 0,42 \\
\hline
\end{tabular}

Tabela 3. Comparação entre os diversos tempos de atravessamento parcial e total.

\begin{tabular}{lrrr}
\hline \multicolumn{1}{c}{$\boldsymbol{\mu}$} & $\mathbf{T L}_{\mathbf{m}}$ & $\mathbf{T L}_{\mathrm{wQ}}$ & $\mathbf{T L}_{\mathbf{w T O}}$ \\
\hline Liberação & 12,61 & 12,58 & 12,61 \\
Corte & 8,70 & 9,43 & 9,19 \\
Costura & 14,75 & 14,72 & 14,66 \\
Montagem & 9,86 & 10,62 & 10,79 \\
Expedição & 9,25 & 9,74 & 9,51 \\
Total & 55,17 & 57,09 & 56,76 \\
\hline
\end{tabular}

da quinta reta, as inclinações são menores, indicando acréscimo de inventário antes da montagem. O acréscimo foi de $152 \%$, como apresentado na última coluna da tabela. A justificativa é que as dezenas de ateliês contratados tiveram no período problemas de gerenciamento, principalmente atrasos, erros de informação e retrabalhos, forçando fluxos de retorno. Foram necessários, na montagem, reparos em materiais que já haviam sido transferidos da costura. A solução deste problema foi uma das ações priorizadas 
Tabela 4. Equações e $\mathrm{R}^{2}$ das retas substitutas.

\begin{tabular}{lcccc}
\hline \multicolumn{1}{c}{ Transição } & \multicolumn{1}{c}{ t } & $\mathbf{R}^{\mathbf{2}}$ & Inventário médio & Aumento $(\%)$ \\
\hline Entrada & $\mathrm{y}=4178,0 \mathrm{x}+22.956$ & 0,9797 & & \\
Fim da liberação & $\mathrm{y}=3784,8 \mathrm{x}-15.173$ & 0,9744 & 32.959 & 316 \\
Fim do corte & $\mathrm{y}=3684,5 \mathrm{x}-43.728$ & 0,9572 & 53.444 & 15 \\
Fim da costura & $\mathrm{y}=3440,8 \mathrm{x}-85.596$ & 0,9444 & 28.288 & 23 \\
Fim da montagem & $\mathrm{y}=2866,9 \mathrm{x}-80.885$ & 0,9408 & 28.920 & 152 \\
Fim da expedição & $\mathrm{y}=2774,4 \mathrm{x}-103.330$ & 0,9141 & & 21 \\
\hline
\end{tabular}

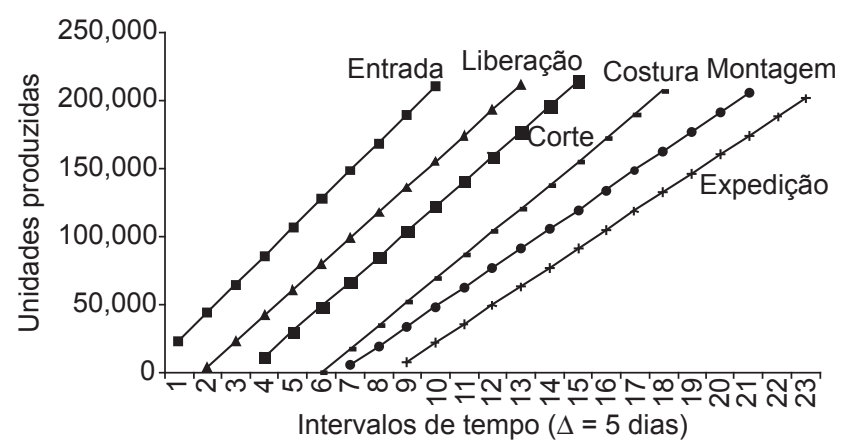

Figura 5. Diagrama de resultado do sistema manufatureiro em estudo.

no controle. Observe que, na indústria estudada, as condições de fornecedores e os requisitos de coleções variam, podendo ocorrer má preparação prévia dos prestadores, como a observada. A eventual ociosidade da montagem, sempre que possível, foi compensada com o adiantamento de remessas liberadas após as quinze estudadas, já que o processo de liberação é contínuo. Este adiantamento, fora do escopo do estudo, criou mais variabilidade no sistema.

\subsection{Medição da variável de controle}

Mediu-se a variável de controle, o tempo de atravessamento total da manufatura, como variável contínua e como intervalo de confiança. Este tempo é a soma de cinco variáveis aleatórias, uma para cada tempo parcial de atividade. Variáveis aleatórias podem ser somadas por ao menos dois métodos: (i) soma das médias e das variâncias, pelo teorema do limite central, com correção dos parâmetros baseada na assimetria e curtose da distribuição soma; e (ii) simulação computacional. No primeiro caso, calculou-se a correlação entre as variáveis para definir o modelo de correção. No caso, usou-se a correção não correlacionada. No segundo caso, conduziram-se testes que identificaram quais modelos de ajustes não puderam ser descartados. Os modelos testados foram: exponencial, gamma, Weibull, normal e lognormal. O modelo exponencial descreve um evento cuja probabilidade condicional é constante. O modelo gamma é o limite para o maior e o Weibull para o menor de $\mathrm{n}$ valores quando $\mathrm{n}$ cresce. $\mathrm{O}$ modelo normal é o limite para um resultado composto pela soma e o lognormal para a multiplicação de n variáveis quando $\mathrm{n}$ cresce (HAHN; SHAPIRO, 1967).

Primeiramente, foi usado o método do teorema do limite central. Usaram-se conjuntos de dez remessas de cada vez: uma remessa concluída sai da amostra e uma iniciada entra na amostra, em uma sistemática de janela móvel de horizonte fixo. Os resultados são apresentados na Tabela 5. Foram usados os $\mathrm{TL}_{\mathrm{m}}$.

Como a convergência ao modelo normal pode não ter se completado, corrigem-se os parâmetros pela assimetria e curtose da distribuição soma. Quanto mais a assimetria $\beta_{1}$ se aproximar de zero e a relação entre a curtose e o quadrado da variância $\beta_{2}$ se aproximar de 3, mais a distribuição se aproxima de uma normal. As expressões de correção surgem em Hahn e Shapiro (1967). Na Tabela 6, apresentam-se os valores originais e corrigidos dos parâmetros, os valores de $\beta 1$ e $\beta 2$ e os intervalos de confiança a $95 \%$ IC $=[\mu \pm 1,96 . \sigma]$ para seis instantes. Os valores esperados não mudaram, mas houve acréscimos nos desvios padrão. O comportamento do valor esperado para o tempo de atravessamento da manufatura surge como variável contínua na Figura 6.

O segundo método foi a simulação computacional.

A liberação de remessas inclui tarefas que se alternam e podem iniciar antes do término da anterior ou ocorrer em paralelo. A costura e a montagem são atividades seqüenciais, pois o produto passa por transformações físicas que exigem que a tarefa anterior se complete antes da próxima tarefa. No corte e na expedição é necessário reunir itens de diversas fontes: o último a chegar conclui a atividade. Foram testados pelo software ProConf 98 ajustes de máxima verossimilhança às distribuições exponencial, Weibull, gamma, lognormal e normal, pelos testes do qui-quadrado e Kolmogorov-Smirnoff. A distribuição de maior verossimilhança correspondeu ao modelo lógico, exceto na expedição, cujo modelo lognormal apresentou um nível de significância maior do que o modelo gamma (9,8 e 9,6\%). Como a diferença é pequena, adotou-se o gamma. Segue o resumo na Tabela 7.

Definidas as distribuições, montou-se um modelo composto pela soma dos cinco tempos parciais. Segundo procedimento apresentado em Law e Kelton (1991), para um nível de confiança de $95 \%$ em que a média se desviará no máximo um dia da média real, com desvio 
Tabela 5. Medição contínua do tempo de atravessamento.

\begin{tabular}{ccccccccccccc}
\hline & \multicolumn{2}{c}{ Liberação } & \multicolumn{2}{c}{ Costura } & \multicolumn{2}{c}{ Corte } & \multicolumn{2}{c}{ Montagem } & \multicolumn{2}{c}{ Expedição } & \multicolumn{2}{c}{ Total } \\
\cline { 2 - 13 } Remessas & $\mu$ & $\sigma$ & $\mu$ & $\sigma$ & $\mu$ & $\sigma$ & $\mu$ & $\sigma$ & $\mu$ & $\sigma$ & $\mu$ & $\sigma$ \\
\hline $1-10$ & 12,66 & 5,2 & 9,05 & 6,3 & 14,02 & 4,3 & 9,21 & 4,6 & 9,96 & 4,6 & 54,90 & 11,3 \\
$2-11$ & 12,13 & 5,6 & 10,25 & 4,6 & 14,52 & 4,6 & 8,56 & 5,1 & 9,63 & 4,6 & 55,09 & 10,9 \\
$3-12$ & 12,40 & 5,7 & 9,65 & 4,5 & 14,42 & 4,5 & 8,11 & 5,2 & 9,43 & 4,7 & 54,01 & 11,1 \\
$4-13$ & 12,33 & 5,7 & 10,08 & 4,7 & 13,92 & 4,7 & 9,66 & 5,9 & 8,93 & 4,4 & 54,92 & 11,5 \\
$5-14$ & 12,12 & 5,6 & 8,78 & 5,0 & 14,22 & 5,0 & 10,37 & 6,3 & 7,53 & 4,1 & 53,02 & 11,7 \\
$6-15$ & 12,63 & 5,5 & 9,30 & 4,1 & 15,44 & 4,1 & 11,57 & 5,5 & 8,53 & 4,4 & 57,47 & 10,7 \\
\hline
\end{tabular}

Tabela 6. Parâmetros contínuos da distribuição do tempo de atravessamento.

\begin{tabular}{cccccccc}
\hline Remessas & $\mu$ original & $\mu$ corrigida & $\sigma$ original & $\sigma$ corrigida & $\beta_{1}$ & $\beta_{2}$ & IC 95\% \\
\hline $1-10$ & 54,90 & 54,90 & 11,3 & 11,3 & 0,0003 & 2,66 & {$[77,05 ; 32,75]$} \\
$2-11$ & 55,09 & 55,09 & 10,9 & 11,5 & 0,0012 & 2,68 & {$[77,63 ; 32,55]$} \\
$3-12$ & 54,01 & 54,01 & 11,1 & 11,5 & 0,0000 & 2,68 & {$[76,55 ; 31,47]$} \\
$4-13$ & 54,92 & 54,92 & 11,5 & 11,7 & 0,0003 & 2,68 & {$[77,85 ; 31,99]$} \\
$5-14$ & 53,02 & 53,02 & 11,7 & 11,8 & 0,0001 & 2,67 & {$[76,15 ; 29,89]$} \\
$6-15$ & 57,47 & 57,47 & 10,7 & 10,8 & 0,0023 & 2,71 & {$[78,64 ; 36,30]$} \\
\hline
\end{tabular}

Tabela 7. Distribuições de máxima verossimilhança ajustadas às atividades.

\begin{tabular}{lccccc}
\hline & & \multicolumn{3}{c}{ Processo } \\
\cline { 2 - 6 } & Liberação & Corte & Costura & Montagem & Expedição \\
\hline Distribuição & normal & gamma & normal & normal & gamma \\
mais verossímil e & $\mu=12,61$ & $\gamma=1,68$ & $\mu=14,75$ & $\mu=9,86$ & $\gamma=2,75$ \\
parâmetros & $\sigma=4,63$ & $\theta=5,38$ & $\sigma=4,13$ & $\sigma=5,44$ & $\theta=3,3632$ \\
& & $t_{0}=0$ & & & $t_{0}=0,2304$ \\
\hline
\end{tabular}

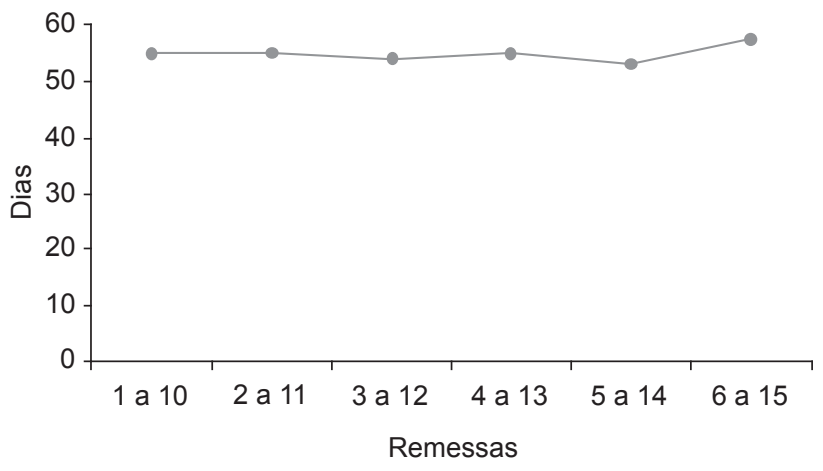

Figura 6. Padrão de comportamento como variável contínua.

padrão de 11,43, foram requeridas 500 replicações. Foi usado o Excel $^{\circledR}$ para a simulação. O desempenho do gerador randômico foi considerado satisfatório, segundo os padrões de aceitação apresentados em Pidd (1998). Foram realizadas 50 rodadas com 500 replicações cada. Os parâmetros médios são apresentados na Tabela 8 . Na Tabela 9 estão as probabilidades de cada atividade deter o maior e o segundo maior tempo de atravessamento (P1 e P2) e a união destas probabilidades, o que aponta potenciais para o controle das atividades. $\mathrm{O}$ resultado
Tabela 8. Parâmetros médios obtidos por simulação computacional.

\begin{tabular}{cccc}
\hline$\mu$ & $\sigma$ & $\beta 1$ & $\beta 2$ \\
\hline 55,71 & 12,16 & 0,0007 & 3,30 \\
\hline
\end{tabular}

Tabela 9. Probabilidades das atividades deterem os maiores tempos de atravessamento.

\begin{tabular}{lccc}
\hline \multicolumn{1}{c}{ Atividade } & P1 (\%) & P2 (\%) & P1 $\cup \mathbf{P 2 ~ ( \% ) ~}$ \\
\hline Liberação & 21,8 & 29,0 & 50,8 \\
Corte & 15,2 & 8,0 & 23,2 \\
Costura & 39,4 & 34,2 & 73,6 \\
Montagem & 11,2 & 16,8 & 28,0 \\
Expedição & 12,4 & 12,0 & 24,4 \\
\hline
\end{tabular}

da simulação, como distribuição do tempo de atravessamento total, segue na Figura 7.

Resumindo, o tempo de atravessamento total, usando o resultado da simulação computacional, tem valor esperado de 55,71 dias, desvio padrão de 12,16 e intervalo de confiança de $95 \%$ dado pela Equação 4. Para controle, este será o resultado da medição.

$$
\left.\mathrm{IC}\right|_{95 \%}=55,71 \pm 1,96 \times 12,16=[79,54 ; 31,88] \text { dias }
$$




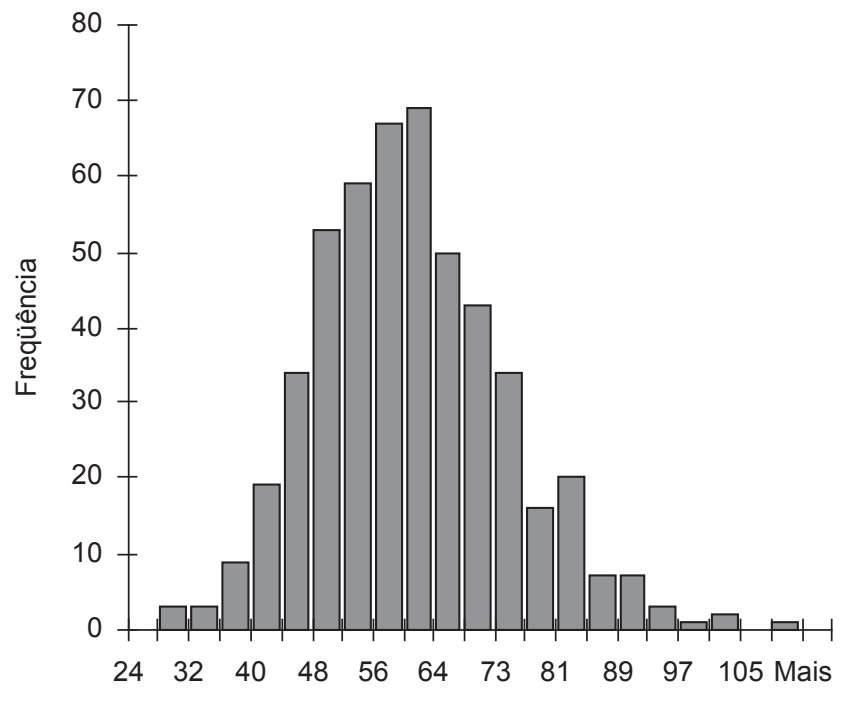

Figura 7. Distribuição do tempo de atravessamento total obtida por simulação.

\subsection{Ações de controle}

O objetivo de entregas da empresa era de $90 \%$ das remessas entregues em até 45 dias. Pela Equação 4, 90\% das entregas ocorriam em até 71 dias. Partiu-se então para melhorar o desempenho. Antes, fizeram-se considerações contextualizadoras: (i) uma vez iniciada, uma ordem deveria ser produzida até a conclusão; (ii) o tempo-padrão de uma ordem deveria ser constante ao longo do tempo; e (iii) o tempo de atravessamento médio, 55,17 dias, foi muito maior do que o tempo-padrão médio, 9,63 dias. Estas considerações simplificam a realidade da manufatura. Nem sempre se inicia a produção tendo em mãos todos os materiais. Por aprendizado, espera-se que as últimas unidades da ordem tenham tempos menores do que as primeiras. Por fim, pode-se considerar que a quantidade produzida pouco interfere no tempo de atravessamento, muito mais influenciado por esperas. Estas considerações podem explicar parte das variabilidades observadas no processo.

Quanto ao sistema, a Figura 4 indicou um desequilíbrio importante: o ritmo de liberação foi maior do que o ritmo de expedição, acumulando inventário e atrasos. Uma alternativa é sincronizar todo o sistema à reta de entradas. A montagem aumentaria a capacidade em $46 \%$, bem como a relação entre os coeficientes angulares das retas de saída e de entrada (4.178/2.867). Outra alternativa é sincronizar todo o sistema à montagem. $\mathrm{O}$ ritmo de entrada seria reduzido em $46 \%$, sobrando capacidade nas demais atividades e realocando pedidos no tempo.

Partiu-se para a definição de ações específicas de controle. Reforça-se que ações não foram inspiradas pela medição, mas por práticas diagnósticas conduzidas pela inteligência que governa a empresa. A medição apenas informa em quais atividades devem-se focar esforços e a sua recorrência informa se as ações foram eficazes.

Como a costura teve os maiores tempos de atravessamento e inventário médio, foi feita uma inspeção diagnóstica na rede de ateliês: (i) a transferência de material entre corte, costura e montagem não era inspecionada quanto à qualidade, apenas quanto à quantidade, surgindo retrabalho durante e após a costura, por uso de materiais inadequados; (ii) ao faltar matéria-prima para concluir uma ordem, o ateliê desativava a equipe ou, para não ficar parado, voltava à fábrica e recolhia material para iniciar outro pedido de outra remessa, mesmo incompleta, gerando excessivos setups e perda de foco; e (iii) muitos ateliês contratavam só após terem ordens em mãos, ocorrendo faltas de pessoal por baixa agilidade na contratação.

Foram planejadas e executadas ações de controle com impacto no sistema de manufatura, mas principalmente nos ateliês: (i) criação de almoxarifado para que as ordens só sejam liberadas para corte e costura após a consolidação dos materiais; (ii) criação de inspeção de qualidade $100 \%$ após o corte e antes da montagem; (iii) redução no número de ateliês, aumentando a escala dos poucos mais qualificados e profissionalizados e eliminando a necessidade de contratar e recontratar pessoal a cada remessa; (iv) redução do lote de transferências da costura para a montagem, para reduzir o acúmulo do inventário observado na medição; e (v) deslocamento de pessoal eventualmente ocioso do corte e da montagem para atividades de costura, na própria fábrica, em paralelo aos ateliês.

Implementadas as ações, coletaram-se dados de 10 remessas, apresentados na Tabela 10. As correlações entre as variáveis continuam distantes de 1 , ou seja, as atividades continuaram independentes. $\mathrm{O}$ novo valor esperado dos tempos de atravessamento é de menos de 29 dias. Os dados comparados antes e depois do controle são apresentados na Tabela 11.

Repetiu-se simulação computacional. Os novos modelos usados foram: liberação (normal), corte (gamma), costura (gamma), montagem (normal) e expedição (gamma). O valor esperado para o tempo até o embarque da remessa foi de 29,31 dias, o desvio padrão de 4,88 e o intervalo de confiança a 95\% o da Equação 5.

$$
\left.\mathrm{IC}\right|_{95 \%}=29,31 \pm 1,96 \times 4,88=[19,75 ; 38,88] \text { dias }
$$

Pela equação, calcula-se que $90 \%$ das entregas passaram a ocorrer antes de 36 dias. Como o objetivo era de 45 dias, conclui-se que o controle do tempo de atravessamento foi bem sucedido. A variabilidade caiu, pois todos os cv caíram. A liberação passou a requerer o maior tempo de atravessamento. Antes, 206.800 pares foram expedidos em 63,3 dias (data da última entrega menos data da primeira entrega), em desempenho médio 
Tabela 10. Segunda coleta de dados. (Fonte: sistema de informações da empresa).

\begin{tabular}{ccccccccc}
\hline $\begin{array}{c}\text { Remessa } \\
\mathbf{n}^{\mathbf{0}}\end{array}$ & $\begin{array}{c}\text { Data } \\
\text { início }\end{array}$ & Quantidade & $\begin{array}{c}\text { TL } \\
\text { liberação }\end{array}$ & $\begin{array}{c}\text { TL } \\
\text { corte }\end{array}$ & $\begin{array}{c}\text { TL } \\
\text { costura }\end{array}$ & $\begin{array}{c}\text { TL } \\
\text { montagem }\end{array}$ & $\begin{array}{c}\text { TL } \\
\text { expedição }\end{array}$ & $\begin{array}{c}\text { TL } \\
\text { total }\end{array}$ \\
\hline 1 & 0 & 11.400 & 9,20 & 3,40 & 5,00 & 3,00 & 4,30 & 24,90 \\
2 & 2,5 & 12.500 & 8,90 & 1,00 & 7,00 & 5,80 & 3,00 & 25,70 \\
3 & 4,0 & 9.500 & 9,80 & 4,00 & 7,00 & 4,30 & 3,50 & 28,60 \\
4 & 8,0 & 17.250 & 11,70 & 4,20 & 6,20 & 8,50 & 3,50 & 34,10 \\
5 & 12,5 & 13.850 & 5,30 & 8,00 & 5,50 & 3,20 & 8,50 & 30,50 \\
6 & 15,5 & 10.500 & 8,70 & 1,50 & 3,40 & 5,20 & 5,20 & 24,00 \\
7 & 21,0 & 17.750 & 11,50 & 5,00 & 7,00 & 8,00 & 5,20 & 36,70 \\
8 & 27,0 & 15.500 & 8,70 & 6,30 & 8,00 & 7,20 & 4,00 & 34,20 \\
9 & 32,0 & 12.150 & 8,90 & 2,20 & 3,80 & 2,80 & 4,50 & 22,20 \\
10 & 36,0 & 13.900 & 8,30 & 6,50 & 4,20 & 5,00 & 3,50 & 27,50 \\
$\mu$ & & & 9,10 & 4,21 & 5,71 & 5,30 & 4,52 & 28,84 \\
$\sigma$ & & & 1,78 & 2,28 & 1,57 & 2,07 & 1,58 & 4,90 \\
cv & & & 0,20 & 0,54 & 0,28 & 0,39 & 0,35 & 0,17 \\
\hline
\end{tabular}

Tabela 11. Tempos de atravessamento antes e depois das ações de controle.

\begin{tabular}{|c|c|c|c|c|c|c|}
\hline & $\begin{array}{c}\text { Média } \\
\text { antes }\end{array}$ & $\begin{array}{l}\text { Média } \\
\text { depois }\end{array}$ & $\begin{array}{c}\text { Redução } \\
\text { na média }(\%)\end{array}$ & cv antes & cv depois & $\begin{array}{r}\text { Redução } \\
\text { no cv }(\%)\end{array}$ \\
\hline Liberação & 12,61 & 9,10 & 27,8 & 0,37 & 0,20 & 46,8 \\
\hline Corte & 8,70 & 4,21 & 51,6 & 0,63 & 0,54 & 13,4 \\
\hline Costura & 14,75 & 5,71 & 61,3 & 0,28 & 0,28 & 1,6 \\
\hline Montagem & 9,86 & 5,30 & 46,2 & 0,55 & 0,39 & 29,3 \\
\hline Expedição & 9,25 & 4,52 & 51,1 & 0,49 & 0,35 & 28,3 \\
\hline Total & 55,17 & 28,84 & 47,7 & 0,21 & 0,17 & 20,0 \\
\hline
\end{tabular}

de 3.266 pares por dia. Após, 134.300 pares foram expedidos em 38,6 dias, em desempenho médio de 3.479 pares por dia. Pela lei de Little (Equação 3), o inventário médio, antes de 180.185 pares, baixou para 100.342 pares. Houve redução do inventário em cerca de $45 \%$, enquanto que o desempenho aumentou em cerca de $6,5 \%$. A exploração deste resultado é remetida à continuidade.

Para complementar a discussão, fez-se o diagrama de resultados para a segunda coleta de dados, após as ações de controle, como apresentado na Figura 8.

É possível observar no diagrama que, apesar da redução tanto do valor esperado como da variabilidade do tempo de atravessamento, o sistema de manufatura ainda não encontrou o equilíbrio. Mais uma vez, é a costura que instabiliza o sistema. Desta vez, a costura foi mais rápida do que os demais processos no atendimento das 10 remessas observadas. Como as retas não se encontraram, não houve ainda interrupção no trabalho, mas houve, no período, consumo de inventário protetivo. Deve-se lembrar que as remessas observadas fazem parte de um fluxo contínuo, pois houve remessas antes e depois das 10 amostradas.

Uma das ações de controle foi reduzir o número de ateliês e concentrar nos restantes mais trabalho, aumentando a escala, reduzindo a ociosidade por falta de

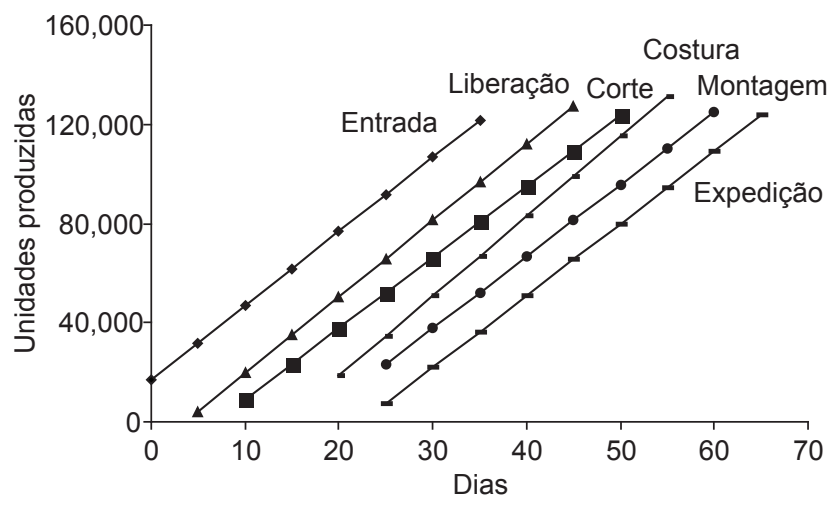

Figura 8. Diagrama de resultados para a segunda coleta de dados.

material e aumentando a eficiência por aprendizado, pois os operadores têm mais quantidade e continuidade de trabalho. Outra ação foi socorrer a costura com pessoal eventualmente ocioso em outros setores. Como as ações parecem ter sido de intensidade maior do que necessário, aumentou-se a probabilidade de haver interrupção por falta de trabalho em alguns prestadores. Talvez seja necessário, na sequiência dos ciclos de controle, reduzir ainda mais o número de ateliês, ajustando a capacidade ao restante do sistema produtivo. Como já colocado, a operação é dispersa geograficamente e, mesmo que a 
conclusão da tarefa tenha sido registrada no sistema de informações, por aproveitamento de rotas, a coleta física pode atrasar. As concentrações resultantes de inventário podem ser de difícil percepção visual, sendo percebidas mais facilmente por informação em meio eletrônico.

\section{Considerações finais}

O objetivo deste artigo foi propor um método para medição e controle do tempo de atravessamento em um sistema de manufatura. Tal controle pode fazer parte de estratégias para aumento de rapidez e de confiabilidade em entregas, em competição baseada no uso do tempo. Por ter relação com o tempo, o inventário em processo na manufatura também foi enfocado. Para teste e refinamento do método, este foi aplicado a um sistema de manufatura calçadista, composto por várias atividades distribuídas geograficamente.

Foram apresentados dois tempos de atravessamento, simples e ponderado, por quantidade ou tempo-padrão da ordem, e um critério para decidir se a variabilidade presente no sistema é suficiente para uso do tempo ponderado. No caso, o teste indicou que o tempo simples era suficiente. De qualquer modo, a base para o uso de tempos ponderados foi apresentada. Também observou-se que alguns sistemas de informações de manufatura usam estimativas fixas para o tempo de atravessamento de uma ordem em um centro de trabalho. Na pesquisa, os tempos se mostraram variáveis aleatórias, tornando impróprio o uso de valores fixos.

Três tipos de variáveis para controle realimentado foram citados: valor instantâneo, valor médio e intervalo de confiança. Para valores instantâneos e médios, apresentou-se o diagrama de resultados e retas substitutas obtidas por mínimos quadrados, que ofereceram expressões analíticas das cargas acumuladas. Pelos coeficientes angulares da primeira e da última reta, foi possível observar que o ritmo da demanda requerida pelos clientes era maior do que o expedido. Para intervalos de confiança, foram necessárias técnicas estatísticas e simulação computacional. Também foi apresentada a lei de Little, da teoria das filas, adaptada à manufatura, para 0 cálculo do inventário médio no sistema.

Aplicado o método para a medição do tempo de atravessamento e verificada sua insuficiência perante o objetivo da empresa, propuseram-se e implantaram-se ações de controle do tempo. A atividade de costura, exercida em rede de prestadores de serviço, foi a que recebeu mais atenção. Ao fim do ciclo de controle, reaplicou-se o método de medição, verificando-se redução no intervalo de confiança para o tempo de atravessamento de ordens e no inventário médio em processo. A redução foi suficiente para cumprir o objetivo de entrega da empresa, mas o sistema continuou em desequilíbrio, ou seja, sem manter o mesmo ritmo de transferências entre as atividades. Mais uma vez, a costura foi a atividade desequilibradora, requerendo ulteriores controles, em ciclo contínuo.

Ao longo dos trabalhos, abriram-se alternativas para a continuidade da pesquisa. Uma alternativa é a extensão do método à outra unidade de valor, o valor financeiro agregado produzido ao longo da manufatura. Como o TLwm é o valor esperado para o tempo que uma unidade do valor leva para atravessar a manufatura, chegar-se-ia a um valor de tempo para que uma unidade de valor financeiro fosse criada, o que pode ser útil em estratégias de competição em TBC. Outra alternativa é a formalização das relações de transformação de arranjos de manufatura do tipo VATX no tipo I, o que permitirá que o método seja estendido a outros arranjos de manufatura. Também cabe investigar se as reduções observadas no tempo de atravessamento e no inventário médio foram relacionadas ao aumento do desempenho da manufatura, e quais ações de controle poderiam ser propostas após a segunda medição. Por fim, acredita-se que a base teórica apresentada possa ser útil para a especificação de um eventual sistema de informação de manufatura que apóie processos de tomada de decisão e controle de produção. 


\title{
Measurement and control of lead-time in a manufacturing system
}

\begin{abstract}
This paper presents a method for the measurement of the lead-time and work-in-process in manufacturing. The measurement was used in an organizational control process in manufacturing, in which the controlled variate was the order lead-time. This kind of control can be useful in manufacture strategies formulated to compete in TBC (timebased competition). We presented the method, who includes elements from the queuing theory, and concerns about simplifying complex manufacturing arrays, in order to facilitate the analysis. In order to test and refine the method, we studied a case in a footwear manufacture system. We collected data from orders and, by statistical techniques and computational simulation, describe how lead-time behaves, in a stochastic way. As the objective was not reached by the measurement, a managerial diagnosis was made, pointing up undesirable effects that addressed control actions. In order to verify the effectivity of the actions, we had new data collected and the measurement method was again applied. This time, the objective of lead-time was reached, showing that the actions were appropriate. Results of the case was analyzed, leading to conclusions and continuities cues.
\end{abstract}

Keywords: Lead-time in manufacturing. Work-in-process in manufacturing. Stochastic processes in manufacturing. Arrays in manufacturing.

\section{Referências bibliográficas}

BARTEZZAGHI, E.; SPINA, G.; VERGANTI, R. Lead-time Models of Business Processes. International Journal of Operations \& Production Management, Bingley, UK, v. 14, n. 5, p. 5-20, 1994.

BECHTE, W. Load-oriented manufacturing control just-in-time production for job shops. Production Planning \& Control, London, v. 5, n. 3, p. 292-307, 1994.

BERTRAND, J.; WORTMANN, J.; WIJNGAARD, J. Production control: a structural and design oriented approach. Amsterdam: Elsevier, 1990. 322 p.

BEN-DAYA, M.; HARIGA, M. Lead-time reduction in a stochastic inventory system with learning consideration. International Journal of Production Research, London, v. 41, n. 3, p. 571-579, 2003.

BONNEY, M. Control of manufacturing systems. Produto e Produção, Porto Alegre, v. 4, n. 1, p. 1-16, 2000.

BREITHAUPT, J.; LAND, M.; NYHUIS, P. The workload control concept: theory and practical extensions of Load Oriented Order Release. Production Planning \& Control, London, v. 13, n. 7, p.625-638, 2002.

BURBIDGE, J. The principles of production control. Plymouth, UK: McDonald \& Evans, 1978. 588 p.

CORREA, H.; GIANESI, I.; CAON, M. Planejamento, Programação e Controle da Produção. S. Paulo: Atlas, 2001. $452 \mathrm{p}$.

COX, J.; SPENCER, M. The constraints management handbook. Boca Raton: St. Lucie Press, 1998. 319 p.

FRIZELLE, G. OPT in perspective. Advanced Manufacturing Engineering, Cambridge, v.1, n.1, p.74-80, 1989.

HAHN, G.; SHAPIRO, S. Statistical models in engineering. N. York: John Wiley \& Sons, 1967. 355 p.

HEARD, E.; PLOSSL, G. Lead-time revisited. Production and Inventory Management Journal, Alexandria, v.25, n.3, p.32-47, 1984.
KLEINROCK, L. Queueing Systems. New York: John Wiley \& Sons, 1975.448 p. v.1.

LAW, A.; KELTON, W. Simulation Modeling and Analysis. New York: McGraw-Hill, 1991. 800 p.

MACCARTHY, B.; FERNANDES, F. A multi-dimensional classification of production systems for the design and selection of production planning and control systems. Production Planning \& Control, London, v.11, n.5, p.481-496, 2000.

PAN, J.; YANG, J. A study of an integrated inventory with controllable lead time. International Journal of Production Research, London, v.40, n.5, p.1263-1273, 2002.

PIDD, M. Computer simulation in management science. Chichester: John Wiley \& Sons, 1998. 279 p.

PLOSSL, G. Production and inventory control. Englewood Cliffs: Prentice Hall, 1985. 448 p.

ROESCH, S. Projetos de estágio e de pesquisa em administração: guia para estágios, trabalhos de conclusão, dissertações e estudos de caso. S. Paulo: Atlas, 1999. 301 p.

SELLITTO, M. Medição e controle de desempenho estratégico em sistemas de manufatura. Porto Alegre, 2005. 195 p. Tese (Doutorado em Engenharia de Produção), Universidade Federal do Rio Grande do Sul - UFRGS.

SLACK, N.; CHAMBERS, S.; HARLAND, C.; HARRISON, A.; JOHNSTON, R. Administração da Produção. S. Paulo: Atlas, 1997. 726 p.

SLACK, N. Vantagem competitiva em manufatura: atingindo competitividade nas operações industriais. S. Paulo: Atlas, 1993. 198 p.

STEKELENBORG, R.; KORNELIUS, L. A diversified approach towards purchasing and supply. In: Walter, C.; Kliemann, F. (org.); Oliveira, J. (org). Productions Management Methods, Proceedings of the IFIP WG5.7. Working conference on evaluation of Productions Management Methods, Gramado, Brazil: Elsevier Science, 1994. 225 p. 
STALK, G.; HOUT, T. Competing against time: how time-based competition is reshaping global markets. New York: Free Pres, 1990. 285 p.

TUBINO, D. Sistemas de Produção: a produtividade no chão de fábrica. Porto Alegre: Bookman, 1999. 182 p.

VOLLMANN, T.; BERRY, W.; WHYBARK, D.; JACOBS, F. Sistemas de planejamento $\&$ controle da produção para o gerenciamento da cadeia de suprimentos. Porto Alegre: Bookman, 2006. 648 p.

WACKER, J. A theoretical model of manufacturing lead times and their relationship to a manufacturing goal hierarchy. Decision Sciences, New York, v.27, n.3, p- 483-518, 1996.

WIENDAHL, H. Load-oriented manufacturing control. Berlin: Springer-Verlag, 1995. $368 \mathrm{p}$.
WIENDAHL, H.; BREITHAUPT, J. Modelling and controlling the dynamics of productions systems. Production Planning \& Control, London, v.10, n.4, p.389-401, 1999.

WIENDAHL, H.; BREITHAUPT, J.: Automatic production control applying control theory, International Journal of Production Economics, Amsterdam, v. 63, n.1, p.33-46, 2001.

WIENDAHL, H.; PETERMANN, D. Production planning and control on the basis of control theory. In: Walter, C.; Kliemann, F.; Oliveira, J. (org). Productions Management Methods, Proceedings of the IFIP WG5.7 Working conference on evaluation of Productions Management Methods. Gramado: Elsevier, 1994. 225 p.

YIN, R. Estudo de caso: planejamento e método. Porto Alegre: Bookman, 2001. 205 p.

\section{Sobre os autores}

\section{Miguel Afonso Sellitto}

Programa de Pós-Graduação em Engenharia de Produção e Sistemas, Universidade do Vale do Rio dos Sinos - Unisinos

Av. Unisinos 950, CEP 93022-000, São Leopoldo, RS, Brasil,

e-mail: sellitto@unisinos.br

\section{Cláudio Walter}

Universidade Federal do Rio Grande do Sul, UFRGS,

Programa de Programa de Pós-Graduação em Engenharia de Produção

Praça Argentina, 9, CEP 90040-020, Porto Alegre, RS, Brasil,

e-mail: maxiprod@pro.via-rs.com.br

Agradecimentos: Deseja-se reconhecer a contribuição dos professores PhD João Luiz Becker, Dr. José Luiz Duarte Ribeiro e Dr. Peter Bent Hansen, membros da banca que julgou a tese de origem deste artigo. 
